\title{
Performance of GaN Power Devices for Cryogenic Applications down to $4.2 \mathrm{~K}$
}

\author{
Luca Nela, Student Member, IEEE, Nirmana Perera, Student Member, IEEE, Catherine Erine, Student \\ Member, IEEE, and Elison Matioli, Member, IEEE
}

\begin{abstract}
Gallium nitride (GaN) power devices are employed in an increasing number of applications thanks to their excellent performance. Nevertheless, their potential for cryogenic applications, such as space, aviation, and superconducting systems, has not yet been fully explored. In particular, little is known on the device performance below liquid nitrogen temperature $(77 \mathrm{~K})$ and the behavior of popular GaN architectures such as Gate Injection Transistor (GIT) and Cascode below room temperature has not yet been reported. Most importantly, it is still unclear how the different device loss contributions, i.e. conduction, soft- and hard-switching losses, change at cryogenic temperatures. In this work, we investigate and compare the performance of four $\mathrm{GaN}$ commercial power devices in a wide temperature range between $400 \mathrm{~K}$ and $4.2 \mathrm{~K}$. All of the tested devices can successfully operate at cryogenic temperature with an overall performance improvement. However, different GaN HEMT technologies lead to significant variations in device gate control and loss mechanisms, which are discussed based on the device structure. The presented results prove the promising potential of the GaN technology for lowtemperature applications and provide precious insights to properly design power systems operating under cryogenic temperatures and maximize their efficiency.
\end{abstract}

Index terms- Gallium Nitride, HEMTs, cryogenic temperature, low-temperature power electronics

\section{INTRODUCTION}

Gallium Nitride (GaN) High-Electron-Mobility Transistors (HEMTs) have emerged as a promising technology for power conversion thanks to their excellent performance [1], [2]. However, while the potential of such devices in power electronic circuits has been the focus of an extensive research effort, typical characterizations occur at room temperature or, at most, up to $150^{\circ} \mathrm{C}$. Yet, numerous applications require the power devices to effectively operate between room temperature down to extreme cryogenic temperatures of $\sim 4 \mathrm{~K}$. Some of the most interesting examples are found in the space and aviation domain [3], [4], and in superconducting machines such as superconducting magnets, energy storage systems and superconducting motors [4]-[6]. While for such applications the power systems are typically thermally insulated and This work was supported in part by the European Research Council under the European Union's H2020 program/ERC Grant Agreement No. 679425, in part by the Swiss National Science Foundation under Assistant Professor (AP) Energy Grant PYAPP2_166901, and in part by the Swiss Office of Energy (SFOE) Grant Number SI501887-01 (MEPCO). Authors are with the Power and Wide-Band-Gap Electronics Research Laboratory, École Polytechnique Fédérale de Lausanne, 1015 Lausanne, Switzerland (e-mail: luca.nela@epfl.ch, nirmana.perera@epfl.ch, catherine.erine@epfl.ch, elison.matioli@epfl.ch).

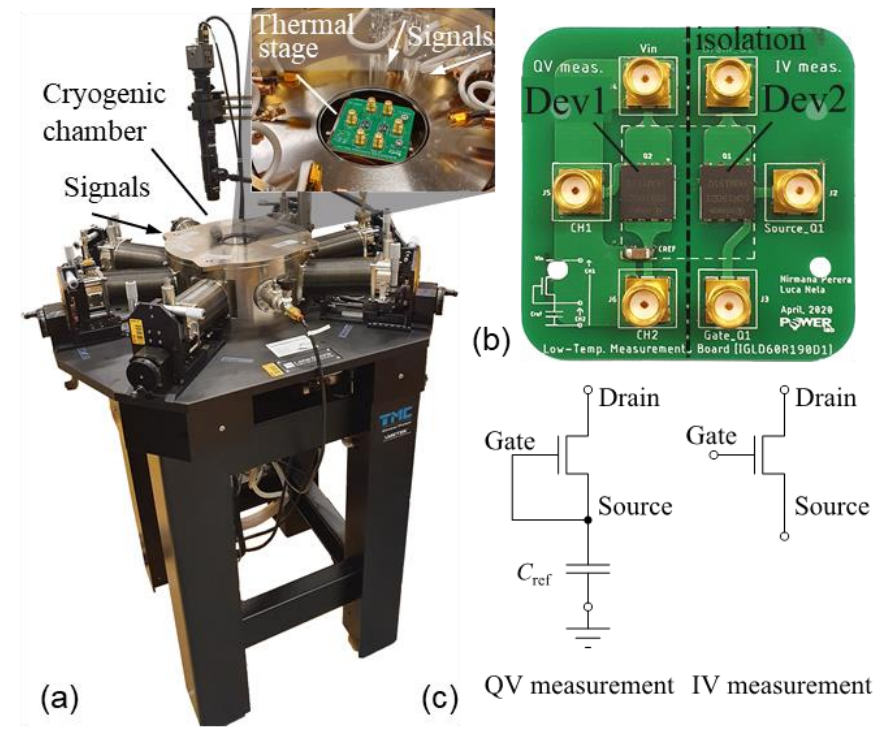

Fig. 1. (a) Cryogenic probe station used for the device characterization. The inset shows the testing PCB mounted on the probe station thermal stage (b) Two identical devices were mounted on the same PCB to allow their switching and DC characterization according to the circuit schematics shown in (c)

maintained at room temperature, this greatly increases their cost, size, and complexity. It would thus be highly beneficial to operate the power converter at cryogenic temperature, which however requires a clear understanding of the power devices' behavior in such conditions.

Typical $\mathrm{Si}$ and $\mathrm{SiC}$ power devices, along with emerging GaN vertical devices, rely on doping of the semiconductor material and show two main effects as the operating temperature is decreased [4], [7], [8]. On the one side, the carrier mobility increases due to the reduced electron-phonon interaction, which is highly beneficial for device performance. On the other side, however, the freeze-out of dopants results in a decrease of the carrier density, leading to a positive shift of the threshold voltage and increased channel resistance. The predominant effect depends on the exact operating temperature but their partial compensation leads to $\mathrm{Si}$ and $\mathrm{SiC}$ power devices that present, depending on the particular device structure, only a mild improvement or even degradation of the low-temperature performance [4]. GaN HEMTs, instead, do not require any doping to achieve a large concentration of electrons in the channel as the carriers are generated by the polarization mismatch between the $\mathrm{AlGaN}$ barrier and the $\mathrm{GaN}$ layer [9]. Since this phenomenon is independent of temperature, GaN HEMTs benefit from the increased carrier 


\begin{tabular}{|c|c|c|c|c|c|c|}
\hline Index & Producer & Name & $\begin{array}{c}\text { Gate } \\
\text { technology }\end{array}$ & $\begin{array}{l}R_{\mathrm{ON}} \\
{[\mathrm{m} \Omega]}\end{array}$ & $\begin{array}{l}V_{\mathrm{DS}} \\
{[\mathrm{V}]}\end{array}$ & $\begin{array}{r}Q_{\text {oss }} \\
{[\mathrm{nC}]}\end{array}$ \\
\hline $\mathrm{T} 1$ & $\begin{array}{c}\text { GaN } \\
\text { Systems }\end{array}$ & $\begin{array}{c}\text { GS66502 } \\
\text { B }\end{array}$ & $\begin{array}{l}\text { Schottky } p \text { - } \\
\text { GaN }\end{array}$ & 200 & 650 & 16 \\
\hline $\mathrm{T} 2$ & Transphorm & $\begin{array}{l}\text { TPH3206 } \\
\text { PSB }\end{array}$ & Cascode & 150 & 650 & 44 \\
\hline $\mathrm{T} 3$ & Panasonic & $\begin{array}{l}\text { PGA26E1 } \\
\text { 9BA }\end{array}$ & $\begin{array}{l}\text { Ohmic } p \text { - } \\
\text { GaN (GIT) }\end{array}$ & 140 & 600 & 17 \\
\hline $\mathrm{T} 4$ & Infineon & $\begin{array}{c}\text { IGLD60R } \\
\text { 190D1 }\end{array}$ & $\begin{array}{l}\text { Ohmic } p \text { - } \\
\text { GaN (GIT) }\end{array}$ & 140 & 600 & 16 \\
\hline
\end{tabular}

Table. 1. Devices investigated in this work and their typical ratings reported in the datasheet. $Q_{\text {oss }}$ is extracted for $V_{\mathrm{DS}}=400 \mathrm{~V}$.

mobility at low temperatures, without suffering any carrier freeze-out.

Despite the promising potential of the GaN technology for cryogenic applications, very few works have investigated the performance of GaN HEMTs in such conditions. In particular, some interesting papers have focused on the characterization of a single commercial device (e.g. GaN Systems [10] and EPC [11]), confirming a significant performance improvement. However, these studies were typically performed down to 77 $\mathrm{K}$, leaving the large temperature range below this value unexplored. Moreover, the low-temperature performance of common GaN HEMT architectures, such as Gate Injection Transistor (GIT) and Cascode, has never been reported, posing questions on their behavior in such conditions. Finally, it is still unclear how the device conduction, soft- and hard-switching losses vary at cryogenic temperature and which contribution becomes dominant.

In this work, we characterize and compare the performance of four $\mathrm{GaN}$ commercial power devices based on the most common device technologies, i.e. $p$-GaN Schottky gate, GIT and Cascode, in the wide temperature range between $400 \mathrm{~K}$ and 4.2 K. The different losses contributions (conduction, hardand soft- switching) as well as the device gate control are analyzed and compared as a function of temperature. Significant variations are observed depending on the technology considered, which are discussed and explained based on the device architecture. The presented results clearly show the potential of GaN power devices at cryogenic temperatures and provide insightful guidelines for the design of power converters operating in such conditions.

\section{EXPERIMENTAL SETUP}

The device characterization was performed in a Lakeshore CPX-VF cryogenic probe station (Fig. 1 (a)). Two identical transistors were soldered on a PCB (Fig. 1 (b)) and mounted on the probe station thermal stage, whose temperature $(T)$ was varied between $400 \mathrm{~K}$ and $4.2 \mathrm{~K}$. Liquid helium refrigerant was used to lower the stage temperature, which was controlled by a PID feedback loop for optimal stabilization within $0.1 \mathrm{~K}$. The measurements were performed under vacuum conditions $\left(10^{-4}\right.$ mbar at $300 \mathrm{~K}$ ) to avoid any condensation. Two transistors were used to provide the device DC and switching characterization simultaneously. The gate and source terminals of one device were shorted and a $10 \mathrm{nF}$ (COG multi-layer ceramic) capacitor $C_{\text {ref }}$ was added to perform Sawyer-Tower
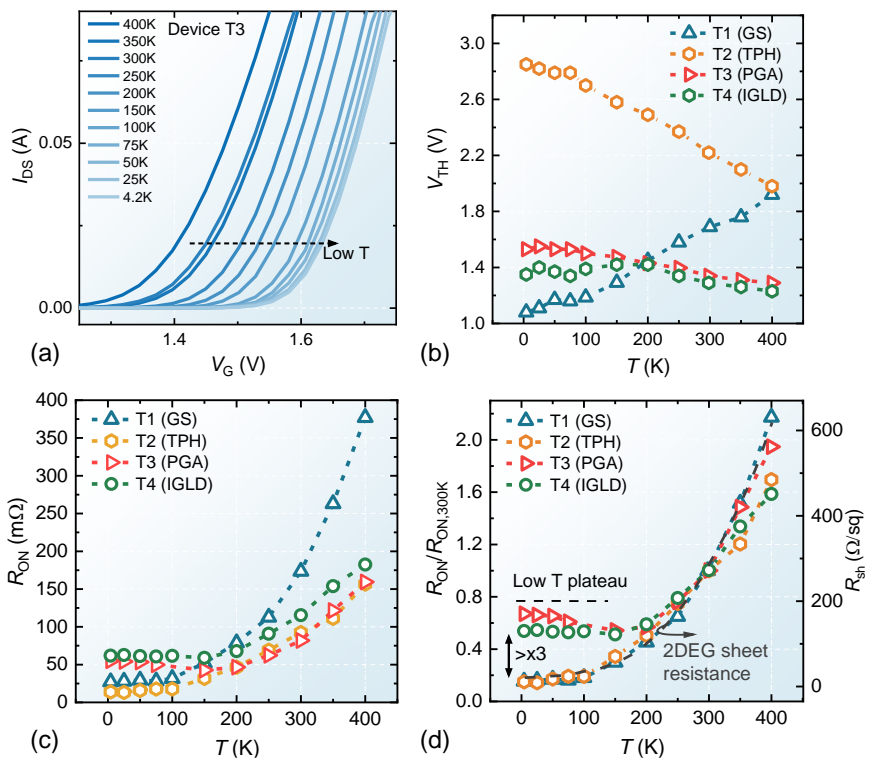

Fig. 2 (a) Transfer characteristics for $\mathrm{T} 3$ from $400 \mathrm{~K}$ to $4.2 \mathrm{~K}$ (b) $V_{\mathrm{TH}}$ as a function of temperature for the four DUTs. $V_{\mathrm{TH}}$ was uniformly defined at a drain-to-source current of $1 \mathrm{~mA}$ (c) Comparison of the devices on-resistance as a function of temperature (d) Normalized $R_{\mathrm{ON}}$ with respect to the room temperature value for the four DUTs. The right $y$-axis shows the typical temperature dependence of the 2DEG sheet resistance $\left(R_{\mathrm{sh}}\right)$.

(ST) measurements [12] to determine the device switching losses (Fig. 1 (c)). The input sinusoidal voltage was provided by a 33600A Keysight waveform generator and amplified by a WMA-300 Falco Systems high-voltage amplifier. The voltage signals were read by an MDO3104 Tektronix oscilloscope. It should be noted that the $C_{\text {ref }}$ value was monitored with temperature and showed no variation. The three terminals of the other transistor were individually connected to a Keysight B1505 analyzer to perform the DC characterization of the device (Fig. 1 (c)). Four commercial devices (DUTs) presenting similar current and voltage ratings but different device technology were characterized and compared (Table 1). While the presented results shed light on the main device trends in a wide temperature range, which depend on the physical properties of the different GaN HEMTs investigated, further statistical analysis on a large number of devices may be necessary to determine precise low-temperature specifications.

\section{RESULTS AND DISCUSSION}

\section{A. Gate Control mechanisms and Conduction losses}

Figure 2 (a) shows the GIT T3 transfer curve as a function of temperature, from which a noticeable positive shift of the characteristic is observable. The device threshold voltage $\left(V_{\mathrm{TH}}\right)$ was extracted at a drain-to-source current $\left(I_{\mathrm{DS}}\right)$ of $1 \mathrm{~mA}$, as suggested in the datasheet, and compared with the one from the other HEMTs technologies. A significant discrepancy between the devices in the value and behavior of $V_{\mathrm{TH}}$ versus $T$ is observed (Fig. 2 (b)). GIT devices (T3 and T4) present a quite constant but slowly increasing $V_{\mathrm{TH}}$ as the temperature is decreased, with the smallest variation among all of the devices, which confirms the excellent $V_{\text {TH }}$ stability of such technology [13]. T2 shows, instead, a large increase of the threshold voltage at cryogenic temperatures, which well agrees with the 


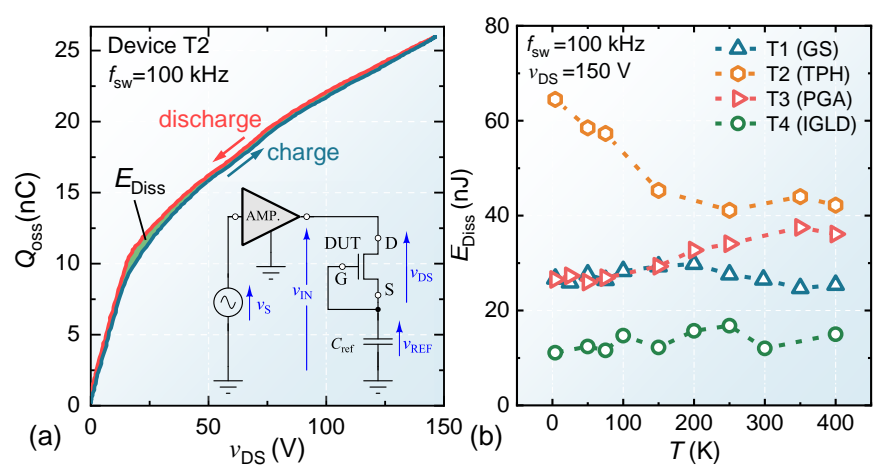

Fig. 3. (a) Sawyer-Tower measurement for T2. The circuit schematics of the measurement setup is shown in the bottom right inset. (b) $E_{\text {Diss }}$ as a function of temperature for the four DUTs.

behavior of the Si device employed to achieve enhancementmode operation. However, thanks to the gate robustness of such architecture, cascode devices can be driven with large gate voltages $\left(V_{\mathrm{G}}\right)$, allowing to compensate the $V_{\mathrm{TH}}$ drift by increasing the driving $V_{\mathrm{G}}$. On the other side, T1 presents a significant $V_{\mathrm{TH}}$ decrease from $1.6 \mathrm{~V}$ at room temperature (RT) down to $\sim 1 \mathrm{~V}$ at $4.2 \mathrm{~K}$. Such effect, which is consistent with Ref. [10], should be carefully considered when designing the gate driving system as the reduced off-state margin may result in dangerous false turn-on events.

Figure 2 (c) illustrates the behavior of the DUTs on-resistance $\left(R_{\mathrm{ON}}\right)$ as a function of temperature. Thanks to the electron mobility $(\mu)$ increase, all of the transistors show a considerable $R_{\mathrm{ON}}$ reduction as the temperature is decreased. This is followed by a plateau region for lower $T$ due to the $\mu$ saturation as a consequence of crystal defects. Yet, while the trend is similar for all of the devices, the amount of such reduction strongly depends on the HEMT technology, which can be better appreciated by considering the normalized $R_{\mathrm{ON}}$ value to the room temperature one (Fig. 2 (d)). T1 and T2 show a very large $R_{\mathrm{ON}}$ decrease of $\sim 6$ times with respect to the RT value, with the onset of the low-temperature plateau region around $100 \mathrm{~K}$. Such a trend agrees well with the sheet resistance $\left(R_{\mathrm{sh}}\right)$ reduction of the $\mathrm{AlGaN} / \mathrm{GaN}$ two-dimensional electron gas (2DEG) as a result of the carrier mobility increase. On the other hand, the two GIT devices present only a $\sim 2$-fold $R_{\text {ON }}$ reduction at cryogenic temperatures and show a premature onset of the low-temperature plateau. This behavior may be due to the introduction of defects in the gate region during the $\mathrm{AlGaN}$ partial recess, which limits the low-temperature mobility. Such diverse behavior among different technologies directly reflects on the device conduction losses and driving circuit, and should be carefully taken into account when choosing the most suitable power transistor for lowtemperature applications.

\section{B. Switching losses}

Depending on the operation conditions of the circuit, power devices are subjected to either soft- or hard- switching losses. While these loss mechanisms are broadly studied at RT conditions, their behavior at cryogenic temperature is yet to be fully investigated.
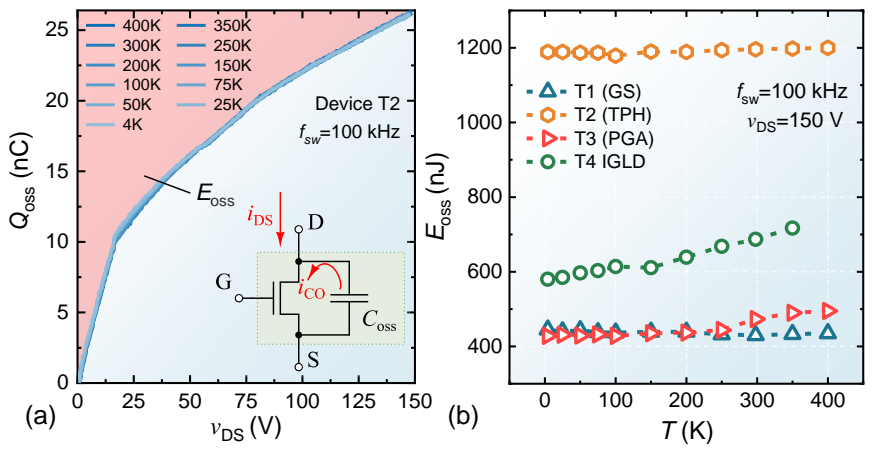

Fig. 4. (a) $E_{\text {oss }}$ extraction from the $Q_{\text {oss }}$ versus $v_{\text {DS }}$ characteristic. In the bottom right inset, the circuit schematics of a device under hard-switching operation is shown. (b) $E_{\text {oss }}$ as a function of temperature for the four DUTs

In particular, soft-switching losses occur due to the non-ideal charging and discharging of the device output capacitance $\left(C_{\text {oss }}\right)$ and become relevant in high-frequency power electronic circuits, such as resonant and quasi-resonant converters [14]. Soft-switching losses are typically characterized by a Sawyer Tower measurement (Fig. 3 (a)), which allows to determine the discrepancies between the $C_{\text {oss }}$ charging and discharging processes. To this end, the transistor source and gate terminals were shorted and a reference linear capacitor $\left(C_{\text {ref }}\right)$ was introduced between the source of the device and the circuit ground. By applying a large-signal sinusoidal input voltage $\left(v_{\mathrm{IN}}\right)$ and measuring the voltage over the reference capacitor $\left(v_{\mathrm{REF}}\right)$, the transistor output charge $\left(Q_{\mathrm{oss}}\right)$ versus drain-to-source voltage $\left(v_{\mathrm{DS}}\right)$ was obtained. It was thus possible to extract the energy dissipated at each switching cycle $\left(E_{\text {Diss }}\right)$ by considering the hysteresis loop between the charging and discharging paths (Fig. 3 (a)). An important feature of this technique is represented by the use of a purely sinusoidal excitation, which avoids the use of signals containing higher frequency harmonics (e.g. square waves). This allows to accurately characterize the DUTs inside the cryogenic chamber at frequencies in the range of several hundreds of $\mathrm{kHz}$ despite the long interconnections required in such measurement.

The value of $E_{\text {Diss }}$ as a function of temperature for four DUTs is shown in Fig. 3 (b), where different trends can be observed. While $\mathrm{T} 1$ shows quite independent behavior in temperature, $\mathrm{T} 3$ presents about a $30 \%$ decrease in $E_{\text {Diss }}$ at $4.2 \mathrm{~K}$ with respect to the RT value. T2 displays, instead, a significant increase of more than $70 \%$ of the RT value. Finally, T4 exhibits relatively small $E_{\text {Diss }}$ values, within the measurement setup accuracy (estimated to be $\pm 5 \mathrm{~nJ}$ ), and thus will not be considered. It should be noted that the variation of the soft-switching losses does not agree with any channel dependencies reported in Fig. 2 . This confirms the recently proposed theories [15]-[17] in which the causes of soft-switching losses are attributed to the $\mathrm{GaN}$ resistive buffer and the $\mathrm{Si}$ substrate, rather than to the channel properties or the presence of stray charges (as for Super Junction Si devices). The consistent $E_{\text {Diss }}$ increase for T2 may be due to the more efficient electron trapping in the $\mathrm{GaN}$ buffer at low temperatures [15]. However, the different behavior among the DUTs suggests that such mechanisms strongly depend on the exact energy level of the trapping sites. Since each manufacturer typically employs a custom epi- 


\begin{tabular}{lcccccc} 
Device & $\begin{array}{c}R_{\mathrm{ON}, \mathrm{RT}} \\
{[\mathrm{m} \Omega]}\end{array}$ & $\begin{array}{c}R_{\mathrm{ON}, 4 \mathrm{~K}} / \\
R_{\mathrm{ON}, \mathrm{RT}}\end{array}$ & $\begin{array}{c}E_{\mathrm{Diss}, \mathrm{RT}} \\
{[\mathrm{nJ}]}\end{array}$ & $\begin{array}{c}E_{\text {Diss,4K}} / \\
E_{\mathrm{Diss}, \mathrm{RT}}\end{array}$ & $\begin{array}{c}E_{\mathrm{oss}, \mathrm{RT}} \\
{[\mathrm{nJ}]}\end{array}$ & $\begin{array}{c}E_{\mathrm{oss}, 4 \mathrm{~K}} / \\
E_{\mathrm{oss}, \mathrm{RT}}\end{array}$ \\
\hline \hline $\mathrm{T} 1$ & 200 & 0.14 & 27 & 1 & 430 & 1 \\
$\mathrm{~T} 2$ & 150 & 0.15 & 42 & 1.73 & 1196 & 1 \\
$\mathrm{~T} 3$ & 140 & 0.66 & 35 & 0.76 & 473 & 0.9 \\
$\mathrm{~T} 4$ & 140 & 0.54 & - & - & 687 & 0.85 \\
\hline \multicolumn{2}{l}{ Table. 2. Comparison of $R_{\mathrm{ON}}, E_{\text {Diss }}$ and $E_{\mathrm{oss}}$ at 300 K and 4.2 K for the DUTs. }
\end{tabular}

structure, the switching loss values and temperature behavior are expected to vary significantly according to the exact $\mathrm{GaN}$ buffer composition and $\mathrm{Si}$ substrate choice. It is however possible to safely assume that soft-switching losses present a weaker temperature dependence with respect to conduction losses.

Hard-switching losses occur when a transistor is switched on while it still holds a large voltage at its drain-to-source terminals. Standard methods to evaluate these losses are based on a double-pulse test (DPT) circuit [18], [19]. Nevertheless, such a technique is very challenging at cryogenic temperatures due to the long wiring required to connect the device inside the cryogenic chamber [10], which has a significant impact on the measurement accuracy. It is however possible to draw important conclusions on the temperature behavior of hardswitching losses by exploiting the presented results, without the use of a DPT measurement.

While hard-switching losses comprise a turn-on and turn-off loss term, turn-off losses can typically be neglected for $\mathrm{GaN}$ devices due to their large transconductance and dv/dt capability [20], [21]. The main hard-switching losses contribution is thus represented by turn-on losses, which can be expressed as a sum of two terms [18], [19]:

$$
P_{s w, h a r d}=f \times E_{o s s}+P_{V I}\left(g_{m}\right)
$$

where $f$ is the switching frequency, $E_{\text {oss }}$ is the energy stored in the transistor output capacitance at a certain off-state $v_{\mathrm{DS}}$ value (Fig. 4 (a)) and $P_{\mathrm{VI}}$ is the loss term associated with the external load current flowing from the circuit through the device channel during the discharging of $\mathrm{C}_{\text {oss. }}$. Figure 4 (a) shows the extraction of the device $E_{\text {oss }}$ values for different temperatures, obtained from the integration of the transistor $Q_{\text {oss }}$ versus $v_{\mathrm{DS}}$ curve. A comparison of $E_{\text {oss }}$ as a function of temperature for the four DUTs is shown in Fig. 4 (b). T1 and T2 present a very constant $E_{\text {oss }}$ value throughout the whole temperature range, which is in agreement with the temperature-independent behavior of the 2DEG concentration. On the other hand, T3 and T4 show a mild $E_{\text {oss }}$ decrease to about $90 \%$ of the RT value at $4.2 \mathrm{~K}$. The contribution of $E_{\text {oss }}$ to hard-switching losses is thus quite constant in a wide temperature range. On the other hand, the second term in (1), $P_{\mathrm{VI}}$, is proportional to the external load current and is linked to the time required to discharge $C_{\text {oss }}$ through the device channel by the current $i_{\mathrm{CO}}$ (inset in Fig. 4 (a)). The discharging time strongly depends on device transconductance $g_{\mathrm{m}}$, which increases significantly with decreasing temperature [18] due to its linear dependence on the carrier mobility (Fig. 2 (c-d)). Hence, while the $E_{\text {oss }}$ contribution is fairly independent of temperature, the value of the $P_{\mathrm{VI}}$ term decreases considerably at cryogenic conditions with a behavior similar to what is shown in Fig. 2 (d). Hard-switching losses thus decrease at low temperatures with a reduction becoming more pronounced as the load current increases.

Overall, some important trends in the different losses contributions at cryogenic temperatures can be identified (Table 2). Conduction losses show the most significant decrease, which is larger $(\sim 6 \mathrm{x})$ for conventional HEMT technologies (T1 and T2) with respect to the GIT devices $(\sim 2 \mathrm{x})$. Soft-switching losses present a weaker temperature dependence without any common trend between the different technologies. For hard-switching losses, while the $E_{\text {oss }}$ component demonstrated to be temperature insensitive, the total loss value decreases due to the enhanced device $g_{\mathrm{m}}$. Thus, while the precise improvement depends on the circuit operation, $\mathrm{GaN}$ power devices show much-enhanced efficiency at cryogenic temperatures.

\section{CONCLUSION}

In this work, we characterized and compared the performance of the most common $\mathrm{GaN}$ commercial power devices in a wide temperature range between $400 \mathrm{~K}$ and $4.2 \mathrm{~K}$. All of the tested devices can successfully operate at cryogenic temperature with an overall performance improvement. However, the different GaN HEMT technologies lead to significant variations in the device gate control and loss mechanisms, which have been explained according to the device structure. The presented results prove the promising potential of the GaN technology for low-temperature applications and provide the circuit designers with precious insights to maximize converters' efficiency in such conditions.

\section{REFERENCES}

[1] H. Amano et al., "The $2018 \mathrm{GaN}$ power electronics roadmap," $J$. Phys. D. Appl. Phys., vol. 51, p. 163001, 2018.

[2] K. J. Chen et al., "GaN-on-Si power technology: Devices and applications," IEEE Trans. Electron Devices, vol. 64, no. 3, pp. 779795, 2017.

[3] H. Gui et al., "Development of High-Power High Switching Frequency Cryogenically Cooled Inverter for Aircraft Applications," IEEE Trans. Power Electron., vol. 35, no. 6, pp. $5670-5682,2020$

[4] H. Gui et al., "Review of Power Electronics Components at Cryogenic Temperatures," IEEE Trans. Power Electron., vol. 35, no. 5, pp. 5144-5156, 2020.

[5] K. Rajashekara and B. Akin, "A review of cryogenic power electronics-status and applications," Proc. 2013 IEEE Int. Electr. Mach. Drives Conf. IEMDC 2013, pp. 899-904, 2013.

[6] C. Jia, "Experimental Investigation of Semiconductor Losses in Cryogenic DC-DC Converters," Thesis, no. February, 2008.

[7] H. Kim, J. Lim, and H. Y. Cha, "DC characteristics of wide-bandgap semiconductor field-effect transistors at cryogenic temperatures," $J$. Korean Phys. Soc., vol. 56, no. 5, pp. 1523-1526, 2010.

[8] I. C. Kizilyalli and O. Aktas, "Characterization of vertical GaN p-n diodes and junction field-effect transistors on bulk GaN down to cryogenic temperatures," Semicond. Sci. Technol., vol. 30, no. 12, p. $124001,2015$.

[9] O. Ambacher et al., "Two-dimensional electron gases induced by spontaneous and piezoelectric polarization charges in $\mathrm{N}$ - and $\mathrm{Ga}$ face $\mathrm{AlGaN} / \mathrm{GaN}$ heterostructures," J. Appl. Phys., vol. 85, no. 6, pp. 3222-3233, 1999.

[10] R. Ren et al., "Characterization and Failure Analysis of 650-V Enhancement-Mode GaN HEMT for Cryogenically Cooled Power Electronics," IEEE J. Emerg. Sel. Top. Power Electron., vol. 8, no. 


\section{IEEE POWER ELECTRONICS LETTER}

1, pp. 66-76, 2020.

[11] J. Colmenares, T. Foulkes, C. Barth, T. Modeert, and R. C. N. Pilawa-Podgurski, "Experimental characterization of enhancement mode gallium-nitride power field-effect transistors at cryogenic temperatures," WiPDA 2016 - 4th IEEE Work. Wide Bandgap Power Devices Appl., pp. 129-134, 2016.

[12] N. Perera et al., "Analysis of Large-Signal Output Capacitance of Transistors using Sawyer-Tower Circuit," IEEE J. Emerg. Sel. Top. Power Electron., vol. 6777, no. c, pp. 1-1, 2020.

[13] H. Li, C. Yao, C. Han, J. A. Brothers, X. Zhang, and J. Wang, "Evaluation of $600 \mathrm{v}$ GaN based gate injection transistors for high temperature and high efficiency applications," WiPDA 2015 - 3rd IEEE Work. Wide Bandgap Power Devices Appl., pp. 85-91, 2015.

[14] M. D. Seeman, "GaN devices in resonant LLC converters: Systemlevel considerations," IEEE Power Electron. Mag., vol. 2, no. 1, pp. 36-41, 2015.

[15] J. Zhuang, G. Zulauf, J. Roig, J. D. Plummer, and J. Rivas-Davila, "An investigation into the causes of coss losses in gan-on-si hemts," 2019 IEEE 20th Work. Control Model. Power Electron. COMPEL 2019, 2019.

[16] M. Guacci et al., "On the Origin of the C oss -Losses in SoftSwitching GaN-on-Si Power HEMTs," IEEE J. Emerg. Sel. Top. Power Electron., vol. 7, no. 2, pp. 679-694, 2019.

[17] J. Zhuang, J. D. Plummer, and J. Rivas-davila, "A physical investigation of large-signal dynamic output capacitance and energy loss in GaN-on-Si power HEMTs at high-frequency applications," 2020 IEEE Energy Convers. Congr. Expo., pp. 189-194, 2020.

[18] E. A. Jones, F. Wang, D. Costinett, Z. Zhang, and B. Guo, "Temperature-dependent turn-on loss analysis for GaN HFETs," Conf. Proc. - IEEE Appl. Power Electron. Conf. Expo. - APEC, vol. 2016-May, pp. 1010-1017, 2016.

[19] R. Hou, J. Lu, and D. Chen, "Parasitic capacitance Eqoss loss mechanism, calculation, and measurement in hard-switching for GaN HEMTs," Conf. Proc. - IEEE Appl. Power Electron. Conf. Expo. - APEC, vol. 2018-March, pp. 919-924, 2018.

[20] G. Deboy, O. Haeberlen, and M. Treu, "Perspective of Loss Mechanisms for Silicon and Wide Band-Gap Power Devices," CPSS Trans. Power Electron. Appl., vol. 2, no. 2, pp. 89-100, 2017.

[21] X. Li, L. Zhang, S. Guo, Y. Lei, A. Q. Huang, and B. Zhang, "Understanding Switching Losses in SiC MOSFET: Toward Lossless Switching," IEEE 3rd Work. Wide Bandgap Power Devices Appl., 2015. 